\title{
Induction of Xa10-like Genes in Rice Cultivar Nipponbare Confers Disease Resistance to Rice Bacterial Blight
}

\author{
Jun Wang, ${ }^{1,2}$ Dongsheng Tian, ${ }^{1}$ Keyu Gu, ${ }^{1}$ Xiaobei Yang, ${ }^{1}$ Lanlan Wang, ${ }^{1}$ Xuan Zeng, ${ }^{1}$ and \\ Zhongchao Yin ${ }^{1,2}$ \\ ${ }^{1}$ Temasek Life Sciences Laboratory, 1 Research Link, National University of Singapore, Singapore 117604, Republic of \\ Singapore; and ${ }^{2}$ Department of Biological Sciences, 14 Science Drive, National University of Singapore, Singapore 117543, \\ Republic of Singapore
}

Accepted 16 March 2017.

Bacterial blight of rice, caused by Xanthomonas oryzae pv. oryzae, is one of the most destructive bacterial diseases throughout the major rice-growing regions in the world. The rice disease resistance $(R)$ gene $X a 10$ confers race-specific disease resistance to $X$. oryzae pv. oryzae strains that deliver the corresponding transcription activator-like (TAL) effector AvrXa10. Upon bacterial infection, AvrXa10 binds specifically to the effector binding element in the promoter of the $R$ gene and activates its expression. $\mathrm{Xa10}$ encodes an executor $\mathrm{R}$ protein that triggers hypersensitive response and activates disease resistance. 'Nipponbare' rice carries two $\mathrm{Xa10}$-like genes in its genome, of which one is the susceptible allele of the $\mathrm{Xa23}$ gene, a Xa10-like TAL effector-dependent executor $R$ gene isolated recently from ' $\mathrm{CBB23}$ ' rice. However, the function of the two Xa10-like genes in disease resistance to $X$. oryzae pv. oryzae strains has not been investigated. Here, we designated the two $\mathrm{Xa10}$-like genes as $\mathrm{Xa10}-\mathrm{Ni}$ and $\mathrm{Xa23}-\mathrm{Ni}$ and characterized their function for disease resistance to rice bacterial blight. Both $\mathrm{Xa10}-\mathrm{Ni}$ and $\mathrm{Xa23}-\mathrm{Ni}$ provided disease resistance to $X$. oryzae pv. oryzae strains that deliver the matching artificially designed TAL effectors (dTALE). Transgenic rice plants containing $\mathrm{Xa10}-\mathrm{Ni}$ and $\mathrm{Xa23}-\mathrm{Ni}$ under the $\mathrm{Xa10}$ promoter provided specific disease resistance to $X$. oryzae pv. oryzae strains that deliver AvrXa10. Xa10-Ni and $\mathrm{Xa23}-\mathrm{Ni}$ knock-out mutants abolished dTALE-dependent disease resistance to $X$. oryzae pv. oryzae. Heterologous expression of $\mathrm{Xa10}-\mathrm{Ni}$ and $\mathrm{Xa23}-\mathrm{Ni}$ in Nicotiana benthamiana triggered cell death. The 19-aminoacid residues at the $\mathrm{N}$-terminal regions of XA10 or XA10-Ni are dispensable for their function in inducing cell death in $N$. benthamiana and the C-terminal regions of XA10, XA10$\mathrm{Ni}$, and XA23-Ni are interchangeable among each other without affecting their function. Like XA10, both XA10-Ni and XA23-Ni locate to the endoplasmic reticulum (ER) membrane, show self-interaction, and induce $\mathrm{ER} \mathrm{Ca}^{2+}$ depletion in leaf cells of $N$. benthamiana. The results indicate that $\mathrm{Xa10}-\mathrm{Ni}$ and $\mathrm{Xa23}-\mathrm{Ni}$ in Nipponbare encode functional executor $R$ proteins, which induce cell death in both monocotyledonous and dicotyledonous plants and have the potential of

Corresponding author: Z. Yin; Telephone: +65 68727420;

E-mail: yinzc@tll.org.sg

*The $\boldsymbol{e}$-Xtra logo stands for "electronic extra" and indicates that two supplementary sequences, twelve supplementary figures, and four supplementary tables are published online.

(c) 2017 The American Phytopathological Society being engineered to provide broad-spectrum disease resistance to plant-pathogenic Xanthomonas spp.

Bacterial blight of rice is one of the most destructive bacterial diseases throughout the major rice-growing regions in the world. The causal agent of bacterial blight of rice is Xanthomonas oryzae pv. oryzae. The $X$. oryzae pv. oryzae strains enter rice leaves, typically through hydathodes at leaf tips and margins or wounds, and multiply in leaf veins and xylem, causing blockage and plant wilting (Niño-Liu et al. 2006). During infection, the $X$. oryzae pv. oryzae strains deliver AvrBs3/PthAlike effectors into host cells via a bacterial type III secretion system (Yang and White 2004). The AvrBs3/PthA-like effectors, also referred to as transcription activator-like (TAL) effectors (Yang et al. 2006), function as transcription factors that enhance the virulence of plant-pathogenic Xanthomonas spp. through the induction of host susceptibility $(S)$ genes (Antony et al. 2010; Cernadas et al. 2014; Hu et al. 2014; Kay et al. 2007; Streubel et al. 2013; Sugio et al. 2007; Yang et al. 2006; Yu et al. 2011; Zhou et al. 2015). The specificity of TAL effectormediated host gene induction is determined by the repetitive central region of each TAL effector, which consists of nearperfect direct repeats of 33- to 35-amino-acid (aa) residues. The two hypervariable amino acids at positions 12 and 13 of each repeat, termed repeat-variable diresidue (RVD), preferentially associate with a different nucleotide to define the length and sequence of effector binding elements (EBE) in the promoters of the targeted host genes (Boch et al. 2009; Moscou and Bogdanove 2009). A few TAL effector-dependent $S$ genes have been identified in rice for susceptibility to $X$. oryzae pv. oryzae and, out of these, the most important and well-studied $S$ genes belong to the MtN3 or SWEET family (Antony et al. 2010; Yang et al. 2006; Yu et al. 2011; Zhou et al. 2015). The SWEET genes encode sugar transporters with seven transmembrane helices and can divert nutrition from the host to facilitate bacterial growth in apoplast of plant cells (Chen et al. 2010).

Plants have coevolved recessive disease resistance $(R)$ genes to overcome this TAL effector-dependent susceptibility. For instance, the recessive mutations in the EBE of SWEET genes in rice abolish the specific recognition between TAL effectors and the cognate SWEET genes and, therefore, cause the failure of $X$. oryzae pv. oryzae to induce SWEET gene expression for pathogen propagation (Chu et al. 2006; Hutin et al. 2015; Zhou et al. 2015). In another example, the recessive allele of the general transcription factor OsTFIIA $\gamma 5$ gene in rice, $x a 5$, provides disease resistance to incompatible $X$. oryzae pv. oryzae strains through the attenuation of TAL effector-dependent 
SWEET gene induction (Huang et al. 2016; Iyer and McCouch 2004). Plants have also coevolved dominant $R$ genes to adapt TAL effector activity to counter bacterial infection (Zhang et al. 2015). The gene products of TAL effector-dependent dominant $R$ genes, referred to as "executor" $R$ genes (Bogdanove et al. 2010), trigger cell-death-associated hypersensitive response (HR) and activate a defense response in plants (Gu et al. 2005; Römer et al. 2007; Strauss et al. 2012; Tian et al. 2014; Wang et al. 2015). Thus far, three TAL effector-dependent dominant $R$ genes have been cloned in rice (Gu et al. 2005; Tian et al. 2014; Wang et al. 2015). $X a 27$ is the first cloned executor $R$ gene in plants that provides broad-spectrum disease resistance to $X$. oryzae pv. oryzae strains ( $\mathrm{Gu}$ et al. 2004, 2005). The XA27 protein is an apoplastic protein that depends on its $\mathrm{N}$-terminal signal-anchorlike sequence to localize to the apoplast (Wu et al. 2008). The susceptible allele of $\mathrm{Xa27}$ ( $x a 27$ ) in 'IR24' rice encodes a protein identical to XA27 (Gu et al. 2005). The induction of $x a 27$ by $X$. oryzae pv. oryzae strains that deliver the artificially designed TAL effector (dTALE) dTALE-xa27, which targets a DNA element in the $x a 27$ promoter, triggered resistance response in IR24 (Li et al. 2013). Following a similar approach, four dTALE genes were designed to induce the four annotated $\mathrm{Xa27}$-like genes in 'Nipponbare' rice but none of the four induced $\mathrm{Xa27}$-like genes conferred resistance to the dTALE-containing $X$. oryzae pv. oryzae strains ( $\mathrm{Li}$ et al. 2013). The Xal0 gene in rice confers race-specific but narrow-spectrum resistance to $X$. oryzae pv. oryzae strains containing TAL effector gene avrXa10 (Hopkins et al. 1992; Yoshimura et al. 1983). The XA10 protein locates to the endoplasmic reticulum (ER) membrane, induces ER $\mathrm{Ca}^{2+}$ depletion, and triggers cell death in rice, Nicotiana benthamiana, and human HeLa cells (Tian et al. 2014). The Xa23 gene is the third executor $R$ gene in rice, which was recently cloned from 'CBB23' (Wang et al. 2015). Xa23 was derived from wild rice (Oryza rufipogon) and confers broad-spectrum resistance to $X$. oryzae pv. oryzae strains (Wang et al. 2014b; Zhang et al. 2001). Xa23 encodes a 113 -aa protein, which shares $50 \%$ identity with XA10 (Wang et al. 2015). By comparing the genetic loci of $X a 10$ and $X a 23$ in the Nipponbare genome, the two $R$ genes were physically mapped to the same locus or adjacent loci on rice chromosome 11 (Gu et al. 2008; Wang et al. 2014a), indicating that the two TAL-dependent $R$ genes may be allelic to each other.

Nipponbare is a typical japonica cultivar that is susceptible to many $X$. oryzae pv. oryzae strains and no bacterial blight $R$ gene has been identified in this cultivar. Two XalO-like genes, Os11g37570 and Os11g37620, were annotated at the corresponding Xa10 locus in the Nipponbare genome. The two genes are 22,177 bp from each other and have the same transcription direction as Xa10, which points toward the centromere (Tian et al. 2014). They encode hypothetical proteins ABA94452 and ABA94457, respectively (Tian et al. 2014). Recently, an alternative open reading frame (ORF) within the predicted ORF of Os11g37620 was found to be the ORF of the susceptible allele of the $X a 23$ gene encoding a protein identical to XA23 (Wang et al. 2015). In this article, we designated Os11g37570 as Xa10$N i$ and the susceptible allele of the $\mathrm{Xa23}$ gene in Nipponbare as $\mathrm{Xa23}-\mathrm{Ni}$ and characterized their function by using approaches of synthetic biology, forward and reverse genetics, plant pathology, as well as cellular biology.

\section{RESULTS}

\section{Induction of $\mathrm{Xa10-Ni}$ or $\mathrm{Xa23-Ni}$ in Nipponbare} confers disease resistance to $X$. oryzae pv. oryzae strains.

dTALE were specifically designed and constructed to induce $\mathrm{Xa10}-\mathrm{Ni}$ or $\mathrm{Xa23}-\mathrm{Ni}$ expression through bacterial inoculation. dTALE-Xa10-Ni targets a DNA element $\left(E B E_{\mathrm{dTALE}-\mathrm{X} 10-\mathrm{Ni}}\right)$ in the Xa1O-Ni promoter (Fig. 1A; Supplementary Fig. S1A;
Supplementary Sequence S1). Disease evaluation showed that Nipponbare was resistant to $X$. oryzae pv. oryzae strain PXO99 ${ }^{A}$ (pHM1dTALE-Xa10-Ni) but was susceptible to the control X. oryzae pv. oryzae strain $\mathrm{PXO}^{\mathrm{A}}$ (pHM1) (Fig. 1B; Supplementary Table S1). Quantitative reverse-transcription polymerase chain reaction (qRT-PCR) analysis also showed that $\mathrm{Xa10}-\mathrm{Ni}$ was specifically induced by $\mathrm{PXO}^{\mathrm{A}}$ (pHM1dTALEXa10-Ni) but not by PXO99 ${ }^{\mathrm{A}}$ (pHM1) (Fig. 1C). The two experiments demonstrated that $\mathrm{Xa1O}-\mathrm{Ni}$ was specifically induced by dTALE-Xa10-Ni and the expression of Xa10-Ni activated disease resistance to PXO99 ${ }^{\mathrm{A}}$ (pHM1dTALE-Xa10-Ni). A 517-bp cDNA of $\mathrm{Xa10-Ni}$ was isolated using $5^{\prime}$ and $3^{\prime}$ rapid amplification of cDNA ends (RACE) and RT-PCR. The XalO-Ni cDNA contains a 12-bp $5^{\prime}$ untranslated region (UTR), a 405-bp ORF, and a 111-bp 3' UTR. The ORF of Xa10-Ni cDNA encodes an XA10-like protein identical to ABA94452 containing 134-aa residues (XA10-Ni).

The TAL effector-dependent gene products were previously found to induce cell death in $N$. benthamiana (Römer et al. 2007; Tian et al. 2014). However, our initial experiment on transient expression of ABA94457 in $N$. benthamiana failed to induce cell death (data not shown). Compared with XA10, ABA94457 has an extended N-terminal region, which is presumably encoded by the predicted exon 1 and part of predicted exon 2 of Os11g37620 (Tian et al. 2014). We suspected that this extended $\mathrm{N}$-terminal region in ABA94457 might not be present in the gene product of the Xal0-like gene. We then designed a dTALE, dTALE-Xa23-Ni-1, by targeting a DNA element $\left(E B E_{\mathrm{dTALE}-\mathrm{Xa23-Ni-1}}\right)$ in the predicted intron 1 of Os11g37620 (Fig. 1A; Supplementary Sequence S2). Disease evaluation demonstrated that Nipponbare was resistant to $\operatorname{PXO}^{\mathrm{A}}$ (pHM1dTALE-Xa23-Ni-1) but not to PXO99 ${ }^{\mathrm{A}}$ (pHM1) (Fig. 1B). The results suggested that a functional $R$ gene was induced by dTALE-Xa23-Ni-1, which triggered disease resistance to PXO99 ${ }^{\mathrm{A}}$ (pHM1dTALE-Xa23-Ni-1). Three independent cDNA (cDNA1, cDNA2, and cDNA3) were isolated by $5^{\prime}$ and $3^{\prime}$ RACE and RT-PCR. After comparing DNA sequences of the three cDNA clones with the genomic sequence at the downstream of $E B E_{\mathrm{dTALE}} \mathrm{Xa23- \textrm {Ni } - 1}$, cDNA1 was found to be derived from the $\mathrm{Xa23}-\mathrm{Ni}$ gene without any intron splicing, whereas cDNA2 and cDNA3 were generated from alternative splicing in the $3^{\prime} \mathrm{UTR}$ or coding region of the $\mathrm{Xa23-Ni}$ gene. A common ORF was identified in cDNA1 and cDNA2, which encodes a 113-aa protein that is identical to XA23 (Wang et al. 2015). For consistency, we designated this 113-aa protein as $\mathrm{XA} 23-\mathrm{Ni}$ in this study. The 5'UTR of cDNA1 of $\mathrm{Xa23-Ni}$ is $237 \mathrm{bp}$ in length and the transcription initiation site is at $85 \mathrm{bp}$ downstream of the $E B E_{\mathrm{dTALE}-\mathrm{Xa23-Ni-1}}$. To confirm the results, we designed a second dTALE, dTALE-Xa23-Ni-2, by targeting an-

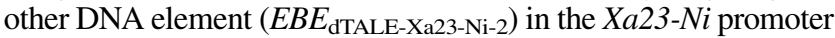
(Fig. 1A). Disease evaluation demonstrated that Nipponbare was resistant to PXO99 ${ }^{\mathrm{A}}$ (pHM1-Xa23-Ni-2) (Fig. 1B). qRT-PCR analysis revealed that $\mathrm{Xa23}-\mathrm{Ni}$ was specifically induced by PXO99 ${ }^{\mathrm{A}}$ (pHM1dTALE-Xa23-Ni-1) and PXO99 ${ }^{\mathrm{A}}$ (pHM1dTALEXa23-Ni-2) but not by PXO99 (pHM1) (Fig. 1D and E).

Pairwise sequence alignment analyses were conducted between the promoters and ORF of the Xa1O, Xa1O-Ni, and Xa23-Ni genes. The identity in the 500-bp promoters analyzed was $41.1 \%$ between $\mathrm{Xa10}$ and $\mathrm{Xa10}-\mathrm{Ni}$ promoters, $44.1 \%$ between $\mathrm{Xa1O}$ and $\mathrm{Xa23}-\mathrm{Ni}$, and $46.4 \%$ between $\mathrm{Xa1O}-\mathrm{Ni}$ and $\mathrm{Xa23}-\mathrm{Ni}$, whereas the identity in the ORF of the three genes was $59.2 \%$ between $X a 10$

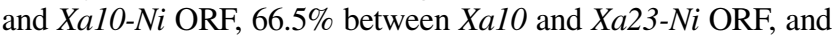
$53.3 \%$ between $\mathrm{Xa10}-\mathrm{Ni}$ and $\mathrm{Xa23}-\mathrm{Ni}$ ORF (Supplementary Figs. S2 to S7). The statistics data indicated that the promoters of Xa10, $\mathrm{Xa10-Ni}$, and $\mathrm{Xa23-Ni}$ are less conserved than their ORF. A similar phenomenon was observed in the previous study, in which the susceptible $\mathrm{Xa23}$ allele in Nipponbare $(\mathrm{Xa23}-\mathrm{Ni})$ and the 
resistant Xa23 allele in CBB23 shared an identical ORF but showed significant difference in the promoter region (Wang et al. $2015)$. Functional $E B E_{\mathrm{AvrXa} 10}$ and $E B E_{\mathrm{AvrXa23}}$ were identified in $\mathrm{Xa10}$ and $\mathrm{Xa23}$ promoters, respectively, which are specifically recognized by AvrXa10 and AvrXa23 (Tian et al. 2014; Wang et al. 2015); however, the two EBE or their consensus sequences were not found in either the Xa10-Ni or Xa23-Ni promoter (Wang et al. 2015).
Transgenic rice plants carrying coding regions of $\mathrm{Xa10}-\mathrm{Ni}$ or $\mathrm{Xa23-Ni}$ under the $\mathrm{Xa10}$ promoter are resistant to $X$. oryzae pv. oryzae strain delivering TAL effector AvrXa10.

TAL effector AvrXa10 binds to $E B E_{\text {AvrXa10 }}$ in the Xa10 promoter and induces $X a 10$ expression, which results in racespecific disease resistance to $X$. oryzae pv. oryzae strains that deliver AvrXa10 (Tian et al. 2014). To further confirm the
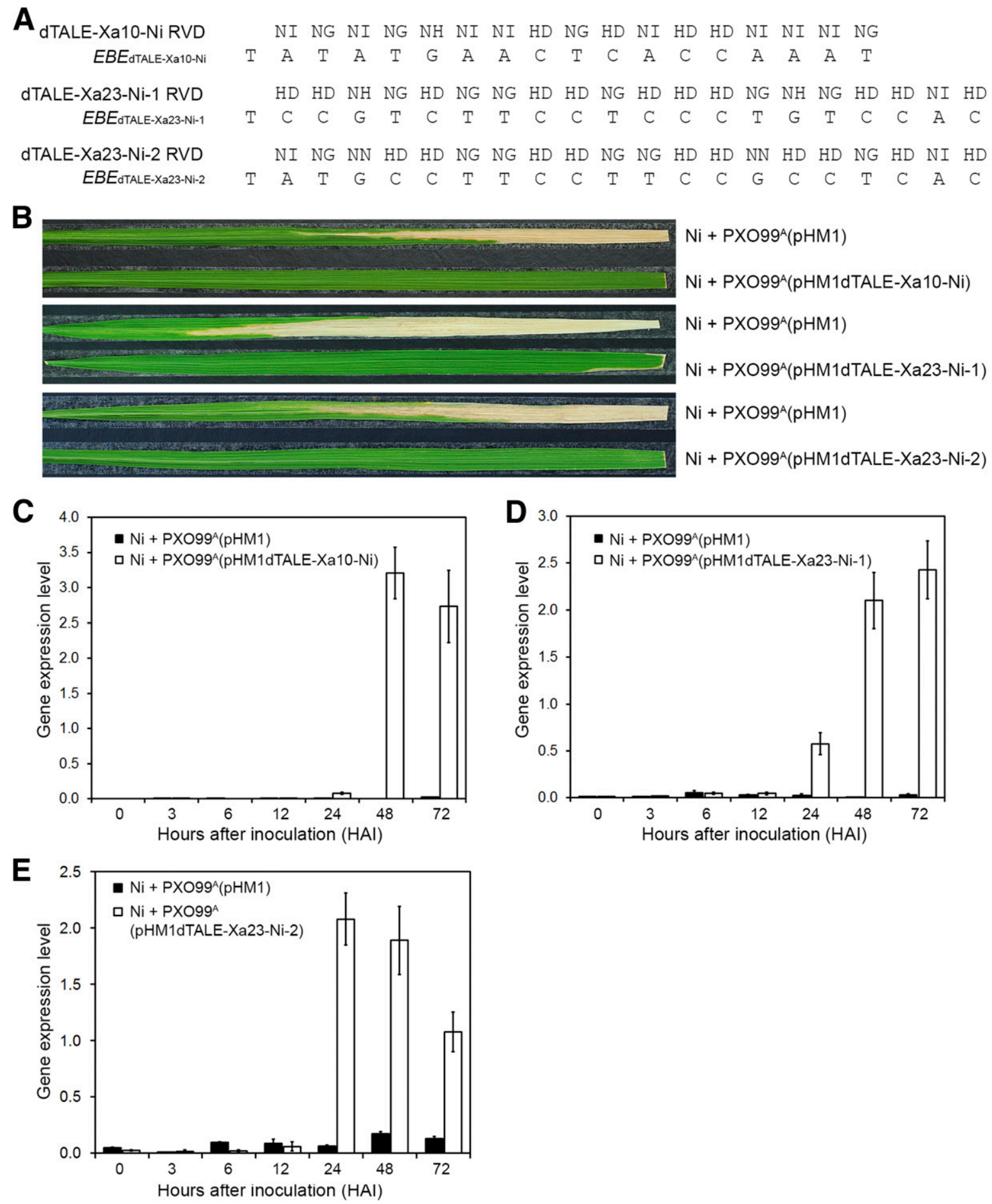

Fig. 1. Designed transcription activator-like effector (dTALE)-induced Xa1O-Ni and Xa23-Ni in Nipponbare conferred disease resistance to Xanthomonas oryzae pv. oryzae. A, Repeat-variable diresidues (RVDs) of dTALEs and DNA sequences of effector binding elements (EBE). EBE start with T at the "0" position. B, Bacterial blight phenotypes of Nipponbare (Ni) at 14 days after inoculation with PXO99 (pHM1), PXO99 (pHM1dTALE-Xa10-Ni), PXO99 ${ }^{\mathrm{A}}$ (pHM1dTALE-Xa23-Ni-1), or PXO99A (pHM1dTALE-Xa23-Ni-2). C, D, and E, Quantitative reverse-transcription polymerase chain reaction analysis of RNA transcripts of $\mathrm{Xa10}-\mathrm{Ni}(\mathrm{C})$ and $\mathrm{Xa23}-\mathrm{Ni}\left(\mathrm{D}\right.$ and E) in Nipponbare (Ni) at different time points after inoculation with PXO99 ${ }^{\mathrm{A}}$ (pHM1), PXO99 ${ }^{\mathrm{A}}$ (pHM1dTALE-Xa10-Ni), PXO99 ${ }^{\mathrm{A}}$ (pHM1dTALE-Xa23-Ni-1), or PXO99 $(\mathrm{pHM1dTALE-Xa23-Ni-2).}$ 
function of $\mathrm{Xa10}-\mathrm{Ni}$ or $\mathrm{Xa23}-\mathrm{Ni}$ in disease resistance, the coding region of Xa10 in its genomic clone SA4671 (Tian et al. 2014) was replaced with the ORF in cDNA of Xa10-Ni or the ORF in cDNA1 of $\mathrm{Xa23}-\mathrm{Ni}$ to generate transcriptional fusion genes $P_{X a 10}: X a 10-N i: T_{X a 10}$ or $P_{X a 10}: X a 23-N i: T_{X a 10}$. The fusion genes were used to produce transgenic plants in Nipponbare genetic background. After bacterial blight inoculation, 18 of 47 transgenic T0 plants of $P_{X a 10}: X a 10-N i: T_{X a 10}$ and 3 of 19 transgenic T0 plants of $P_{X a 10}: X a 23-N i: T_{X a 10}$ were resistant to PXO99 (pHM1avrXa10) (Supplementary Table S2). In addition, the specific resistance of $P_{X a 10}: X a 10-N i: T_{X a 10}$ or $P_{X a 10}: X a 23-N i$ : $T_{X a 10}$ to $\mathrm{PXO}^{\mathrm{A}}{ }^{\mathrm{A}}$ (pH1avrXa10) but not to $\mathrm{PXO}^{\mathrm{A}}{ }^{\mathrm{A}}$ (pH1) was detected in the T1 generation (Fig. 2). These results demonstrated that both $P_{X a 10}: X a 10-N i: T_{X a 10}$ and $P_{X a 10}: X a 23-N i$ : $T_{X a 10}$ are functional in conferring disease resistance to $\mathrm{PXO} 99^{\mathrm{A}}$ (pHM1avrXa10). In another experiment, the $X a 10$ coding region was replaced with the ORF in cDNA3 of $\mathrm{Xa23-Ni}$ (ORF2), which encodes a hypothetical protein with 70 -aa residues, to generate $P_{X a 10}: X a 23-N i-O R F 2: T_{X a 10}$ gene. Seventy-five T0 plants of $P_{X a 10}: X a 23-N i-O R F 2: T_{X a 10}$ were produced; however, none of transgenic T0 plants was resistant to $\mathrm{PXO}^{\mathrm{A}}{ }^{\mathrm{A}}$ (pHM1avrXa10) (Supplementary Fig. S8). The result indicated that the 70-aa protein encoded by the ORF2 in cDNA3 of Xa23-Ni does not provide disease resistance to $X$. oryzae pv. oryzae.

\section{Xa10-Ni or Xa23-Ni knock-out mutants abolishes disease resistance to $X$. oryzae pv. oryzae.}

The knock-out mutants of $\mathrm{Xa1O}-\mathrm{Ni}$ or $\mathrm{Xa23}-\mathrm{Ni}$ were generated using transcription activator-like effector nuclease (TALEN) or clustered regularly interspaced short palindromic repeats (CRISPR)-associated protein-9 nuclease (CRISPR/Cas9) technology (Li et al. 2012; Ma et al. 2015). Four independent knockout mutants of $\mathrm{Xa10}-\mathrm{Ni}$ were generated using TALEN technology (Fig. 3A). They had a 4- to 14-bp deletion in the interspace region between two TALEN target sites in the coding region of $\mathrm{Xa1O}-\mathrm{Ni}$, which resulted in frame-shift mutation (Fig. 3A). Three independent knock-out mutants of $\mathrm{Xa23}-\mathrm{Ni}$ were produced using CRISPR/Cas9 technology (Fig. 3A). They had either 1-bp insertion or 17-bp deletion in the gRNA target site (Fig. 3A). Disease evaluation demonstrated that the Xa10-Ni knock-out mutants were susceptible to PXO99 ${ }^{\mathrm{A}}$ (pHM1dTALE-Xa10-Ni) and the $\mathrm{Xa23}-\mathrm{Ni}$ knock-out mutants were susceptible to PXO99 ${ }^{\mathrm{A}}$ (pHM1dTALE-Xa23-Ni-2) (Fig. 3B; Supplementary Table S3). qRT-PCR analysis indicated that the mutated $\mathrm{Xa1O}-\mathrm{Ni}$ or $\mathrm{Xa23}-\mathrm{Ni}$ gene was still specifically induced by $\mathrm{PXO}^{\mathrm{A}}{ }^{\mathrm{A}}$ that delivered the matching dTALE (Fig. 3C and D). Nine XalO-Ni and $\mathrm{Xa23-Ni}$ double knock-out mutants were generated by genetic crossing (Table 1). They were susceptible to both PXO99 ${ }^{\text {(pHM1dTALE- }}$
Xa10-Ni) and PXO99 ${ }^{\mathrm{A}}$ (pHM1dTALE-Xa23-Ni-2) (Supplementary Fig. S9). It was noted that all of the double knock-out mutants had normal morphological phenotypes in growth and development (data not shown). The results indicated that, except for functioning in disease resistance, $\mathrm{Xa1O}-\mathrm{Ni}$ or $\mathrm{Xa23}-\mathrm{Ni}$ might not be required for normal plant growth and development.

\section{XA10-Ni and XA23-Ni induce} cell death in $N$. benthamiana.

XA10-Ni and XA23-Ni were transiently expressed in leaf cells of $N$. benthamiana via Agrobacterium tumefaciens-mediated infiltration. Both XA10-Ni and XA23-Ni were able to induce cell death at the infiltrated area on leaves of $N$. benthamiana (Fig. 4). The early cell death with the damage of cell membrane in leaves of $N$. benthamiana could be detected by trypan blue staining at $24 \mathrm{~h}$ after infiltration (HAI) (Fig. 4). N. benthamiana infiltrated with $A$. tumefaciens harboring an empty vector only left infiltration marks due to mechanical wounding (Fig. 4). Compared with XA23-Ni, both XA10 and XA10-Ni have an extended 19-aa N-terminal region (Fig. 5A). Based on the results of transmembrane helix prediction using the SOSUI program (http: //harrier.nagahama-i-bio.ac.jp/sosui/sosui_submit.html), this 19aa $\mathrm{N}$-terminal region belongs to the first transmembrane helix in XA10 or XA10-Ni (Supplementary Fig. S10). The XA10 mutant with this N-terminal region removed (D19XA10) still caused cell death in $N$. benthamiana (Fig. 5B). The XA10-Ni mutant with the 19-aa N-terminal region deleted (D19XA10-Ni) slightly delayed its induction of cell death in $N$. benthamiana (Fig. 5B). XA10, XA10-Ni, and XA23-Ni have a short hydrophilic C-terminal regions (Fig. 5A). Mutation in the aspartic acid/glutamic acid (DE)rich motif of XA10, such as XA10C113T and XA10NQ, abolished cell death induction in $N$. benthamiana and disease resistance in rice (Tian et al. 2014). Domain swapping for the C-terminal regions was conducted between members of the three XA10-like proteins. The resulted hybrid proteins (nXA10-cXA10-Ni, nXA10-cXA23Ni, nXA10-Ni-cXA10, nXA10-Ni-cXA23-Ni, nXA23-Ni-cXA10, and nXA23-Ni-cXA10-Ni) were still able to induce cell death in $N$. benthamiana (Fig. 5B). The results demonstrated that the 19-aa $\mathrm{N}$-terminal regions in XA10 and XA10-Ni are dispensable and the short hydrophilic C-terminal regions of XA10-like proteins are functionally conserved and interchangeable.

\section{XA10-Ni and XA23-Ni locate to the ER membrane and trigger ER calcium depletion.}

The subcellular localization of XA10-Ni and XA23-Ni were detected by coexpressing enhanced yellow fluorescent protein (eYFP)-RcDGAT2, an ER membrane marker (Tian et al. 2014), with functional XA10-Ni-enhanced cyan fluorescent protein

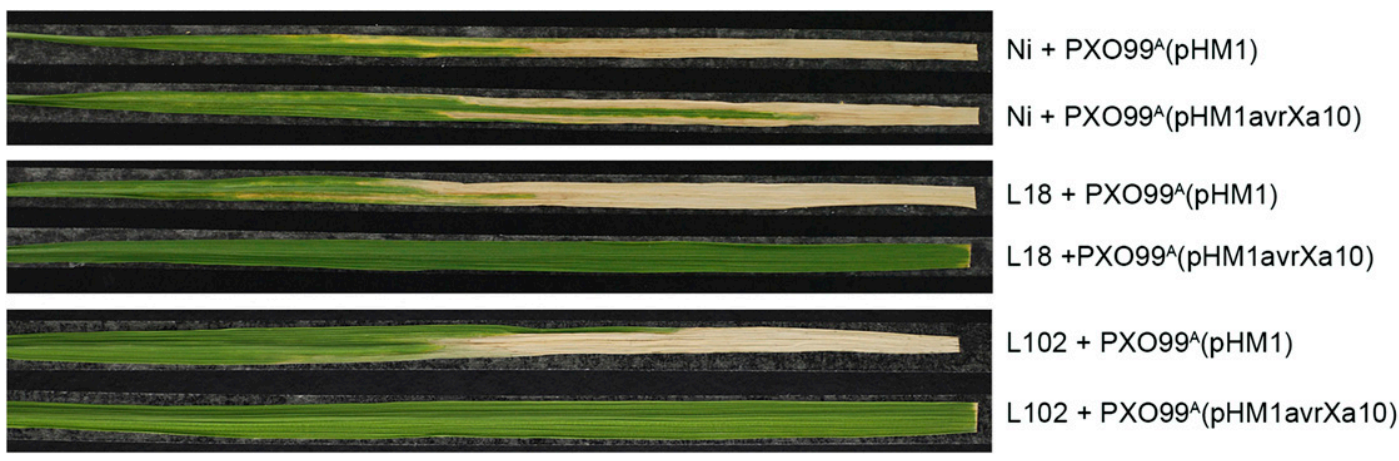

Fig. 2. Bacterial blight phenotypes of transgenic plants carrying $P_{X a 10}: X a 10-N i: T_{X a 10}$ or $P_{X a 10}: X a 23-N i: T_{X a 10}$. Leaves of Nipponbare and transgenic T1 plants were photographed at 14 days after inoculation with PXO99 ${ }^{\mathrm{A}}(\mathrm{pHM})$ and PXO99 $(\mathrm{pHM} 1 \mathrm{avrXa10}) . \mathrm{Ni}=\mathrm{Nipponbare}, \mathrm{L} 18=\operatorname{line} 18$ of $P_{X a 10}: X a 10-N i: T_{X a 10}$, and L102 = line 102 of $P_{X a 10}: X a 23-N i: T_{X a 10}$. 
(eCFP) or XA23-Ni-eCFP in leaf cells of $N$. benthamiana. Both XA10-Ni-eCFP and XA23-Ni-eCFP were colocalized with eYFP-RcDGAT2 to the ER membrane in leaf epidermal cells of $N$. benthamiana (Fig. 6). In addition, XA10-Ni and XA23-Ni showed self-interaction in a bimolecular fluorescence complementation (BiFC) assay (Fig. 7). The fluorescent signal from the N-terminal region of YFP (nYFP)-XA10-Ni/C-terminal region of YFP (cYFP)-XA10-Ni or nYFP-XA23-Ni/cYFP$\mathrm{XA} 23-\mathrm{Ni}$ interactions had localization patterns on the nuclear envelope and the ER membrane similar to those of XA10-NieCFP or XA23-Ni-eCFP alone (Fig. 7). No signal was detected in the control experiments for the interaction between nYFP and cYFP-XA10-Ni or between nYFP and cYFP-XA23-Ni (Fig. 7). The results indicated that both XA10-Ni and XA23-Ni locate to the ER membrane and form oligomers through selfinteraction. ER is one of the important intracellular $\mathrm{Ca}^{2+}$ stores in eukaryotic cells. To check whether XA10-Ni and XA23-Ni had a function similar to that of XA10 in inducing $\mathrm{Ca}^{2+} \mathrm{de}-$ pletion from ER (Tian et al. 2014), XA10-Ni-mCherry or
XA23-Ni-mCherry was transiently coexpressed with the ER luminal $\mathrm{Ca}^{2+}$ indicator YC4.60ER or cytosol $\mathrm{Ca}^{2+}$ indicator YC3.60 in $N$. benthamiana. The ER $\mathrm{Ca}^{2+}$ concentrations in the XA10-Ni-mCherry- and XA23-Ni-mCherry-expressing cells, measured by the emission ratio of fluorescence resonance energy transfer (FRET)/CFP of YC4.60ER, were 41.3 and $48.6 \%$

Table 1. $\mathrm{Xa10}-\mathrm{Ni}$ and $\mathrm{Xa23}-\mathrm{Ni}$ double knock-out mutants

\begin{tabular}{lll}
\hline Mutant line & \multicolumn{1}{c}{ Mutation in $\mathbf{X a 1 0 - N i}$} & Mutation in $\mathbf{X a 2 3 - N i}$ \\
\hline L1 & ATTGCGATTGTCAT, deletion & $\mathrm{T}$, insertion \\
L2 & ATTGCGATTGTCAT, deletion & A, insertion \\
L3 & ATTGCGATTGTCAT, deletion & C, insertion \\
L4 & ATTGCGATTGTCAT, deletion & $\mathrm{G}$, insertion \\
L5 & ATAT, deletion & $\mathrm{C}$, insertion \\
L6 & GCGAT, deletion & $\mathrm{G}$, insertion \\
L7 & GCGAT, deletion & T, insertion \\
L8 & GCGAT, deletion & A, insertion \\
L9 & GCGAT, deletion & CCGG, deletion \\
\hline
\end{tabular}

A

Rice lin

Nipponbare

Xa10-Ni KO L31

Xa10-Ni KO L36

Xa10-NiKO L53

Xa10-Ni KO L61

Nipponbare

Xa23-Ni KO L131

Xa23-Ni KO L136

Xa23-Ni KO L162
DNA sequence

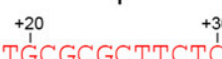

TGCGCGCTTCTCCTCATCGCCGI TGCGCGCTTCTCCTCATCGCCGTCCAT-----GATTGTCATCTCCTGCCCGGAGCCA TGCGCGCTTCTCCTCATCGCCGTCCAT------------CTCCTGCCCGGAGCCA TGCGCGCTTCTCCTCATCGCCGTCC----TGCGATTGTCATCTCCTGCCCGGAGCCA TGCGCGCTTCTCCTCATCGCCGTCCATATT-----TGTCATCTCCTGCCCGGAGCCA $+20$ $+30$ $+40$ $+50$ TCAAGGÀGCTGGCAGCC'GTAG-CCGGTÀTACACATGATCCTCATCTA'CC TCAAGGAG-----------A-------ATACACATGATCCTCATCTACC TCAAGGAGCTGGCAGCCGTAGTCCGGTATACACATGATCCTCATCTACC TCAAGGAGCTGGCAGCCGTAGACCGGTATACACATGATCCTCATCTACC

\section{Mutation}

Wt

$-5$

$-14$

$-4$

$-5$

\section{$-17$}

$+\mathrm{T}$

$+\mathrm{A}$

B
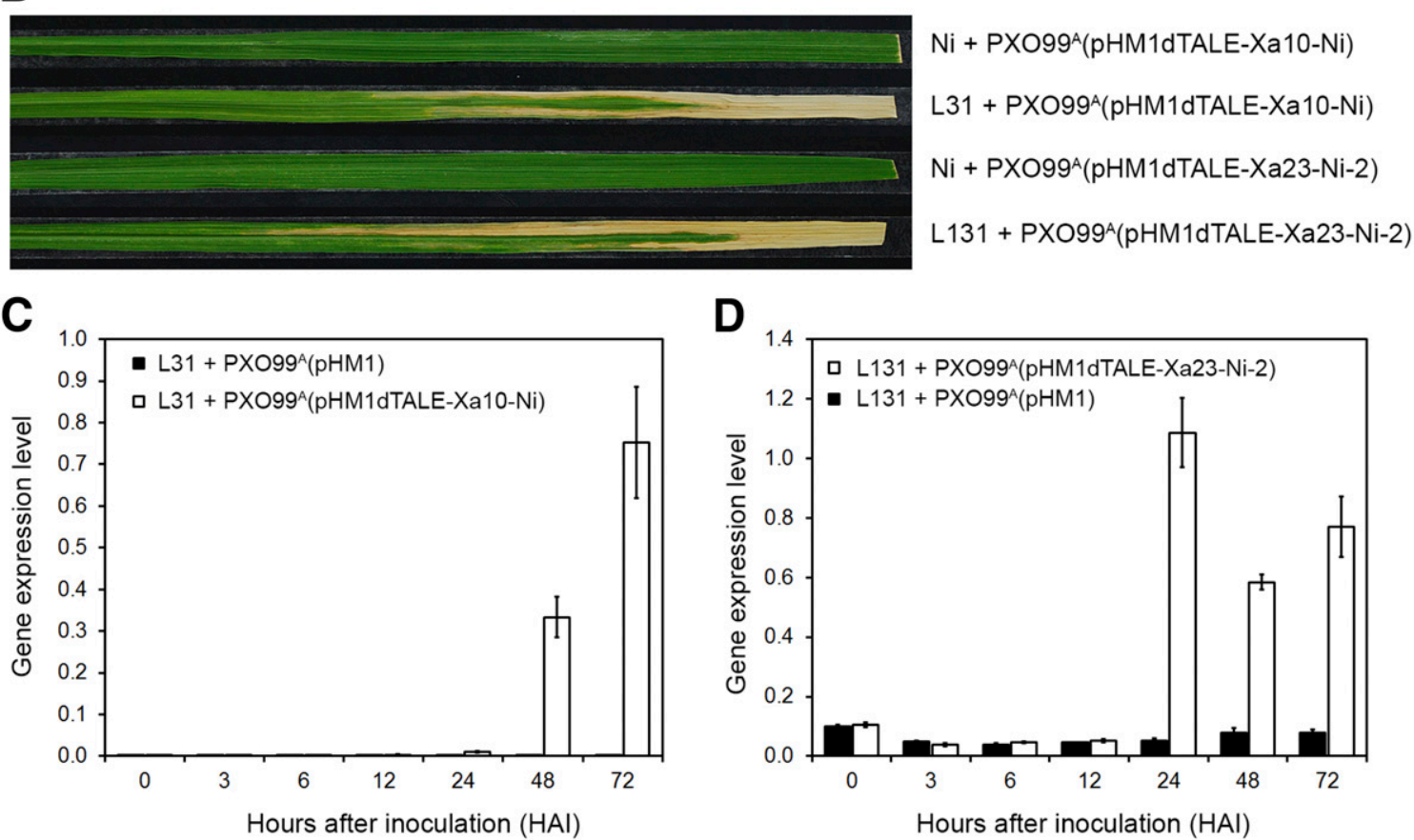

Fig. 3. Disease resistance was abolished in Xa10-Ni and Xa23-Ni knock-out mutants. A, Alignment of DNA sequences in the coding regions between Nipponbare and $\mathrm{Xa1O}-\mathrm{Ni}$ or $\mathrm{Xa23}-\mathrm{Ni}$ knock-out mutants. The DNA-binding positions of the paired transcription activator-like effector nucleases in the $\mathrm{Xa1O}-\mathrm{Ni}$ coding region are shown in red, whereas the target DNA sequences of gRNA of CRISPR/Cas9 in Xa23-Ni coding region are displayed in blue; "“" was introduced to achieve maximal alignment. The nucleotide "A" in starting codon "ATG" was marked as " +1 ". Wt = wild-type. B, Bacterial blight phenotypes of Nipponbare (Ni), Xa10-Ni knock-out mutant L31, and Xa23-Ni knock-out mutant L131 at 14 days after inoculation with PXO99 (pHM1dTALE-Xa10-Ni) or PXO99 ${ }^{\mathrm{A}}$ (pHM1dTALE-Xa23-Ni-2). C and D, Quantitative reverse-transcription polymerase chain reaction analysis of RNA transcripts in Xa10-Ni knock-out mutant L31 (C) and Xa23-Ni knock-out mutant L131 (D) at different time points after inoculation with PXO99 ${ }^{\mathrm{A}}$ (pHM1) (C and D), PXO99 ${ }^{\mathrm{A}}$ (pHM1dTALEXa10-Ni) (C), or PXO99 (pHM1dTALE-Xa23-Ni-2) (D). 
lower, respectively, than that in the control mCherry-expressing cells (Fig. 8). Meanwhile, the cytosolic $\mathrm{Ca}^{2+}$ concentration in the XA10-Ni-mCherry- and XA23-Ni-mCherry-expressing cells, measured by the emission ratio of FRET/CFP of YC3.60, were 123.1 and $77.6 \%$ higher, respectively, than that in the control mCherry-expressing cells (Fig. 8). The results indicated that the ER $\mathrm{Ca}^{2+}$ depletion in the $N$. benthamiana cells, which is accompanied by cytosolic $\mathrm{Ca}^{2+}$ increase, is associated with the expression of XA10-Ni-mCherry or XA23Ni-mCherry.

\section{DISCUSSION}

The TAL effector-dependent executor $R$ genes rely on their promoters to perceive the presence of corresponding TAL effectors from bacteria, whereas their gene products perform defense function when they are expressed (Gu et al. 2005; Römer et al. 2007; Strauss et al. 2012; Tian et al. 2014; Wang et al. 2015). In this study, we have characterized two Xa10-like executor $R$ genes, $\mathrm{Xa1O}-\mathrm{Ni}$ and $\mathrm{Xa23}-\mathrm{Ni}$, in Nipponbare rice using forward and reverse genetics together with synthetic biology. The induction of $\mathrm{Xa1O}-\mathrm{Ni}$ and $\mathrm{Xa23}-\mathrm{Ni}$ in Nipponbare provided disease resistance to $X$. oryzae pv. oryzae strains that deliver the matching dTALE. The ORF of $\mathrm{Xa1O-Ni}$ or $\mathrm{Xa23-Ni}$ could be used to replace the coding sequence in the $\mathrm{Xa10}$ gene and the resulting hybrid genes showed AvrXa10-dependent disease resistance specificity, indicating that both $\mathrm{Xa10-Ni}$ and $\mathrm{Xa23}-\mathrm{Ni}$ can function as executor $R$ genes in a TAL effector-dependent manner. Knock-out of $\mathrm{Xa10-Ni}$ or Xa23-Ni in Nipponbare abolished dTALE-dependent and $\mathrm{XA} 10-\mathrm{Ni}^{-}$or $\mathrm{XA} 23-\mathrm{Ni}-$ mediated disease resistance to $X$. oryzae pv. oryzae. Similar to the XA10 protein, both XA10-Ni and XA23-Ni are transmembrane proteins that locate to the ER membrane, trigger $\mathrm{ER} \mathrm{Ca}^{2+}$ depletion and cell death, and function broadly in both monocotyledonous and dicotyledonous plants. Considering that $\mathrm{Xa10}, \mathrm{Xa1O}-\mathrm{Ni}, \mathrm{Xa23}-\mathrm{Ni}$, and $\mathrm{Xa23}$ locate to the same genetic locus or adjacent genetic loci, they form a family of Xa10-like executor $R$ genes that encode ER membrane-localized executor $\mathrm{R}$ proteins for triggering cell death or $\mathrm{HR}$ and activating disease resistance.

XA10-like executor $\mathrm{R}$ proteins are conserved in structure and function. XA23 and XA23-Ni have three predicted transmembrane helices, whereas XA10 and XA10-Ni have an extended $\mathrm{N}$-terminal region forming a putative additional transmembrane helix. Deletion of this N-terminal transmembrane helix in XA10 or XA10-Ni did not affect their function in induction of cell death. In addition, the short hydrophilic C-terminal regions of XA10-like proteins are interchangeable. Like XA10, both XA10-Ni and XA23-Ni/XA23 locate to the ER membrane and show self-interaction. They also induce the depletion of free $\mathrm{Ca}^{2+}$ from the ER to cytoplasm in leaf epidermal cells of $N$. benthamiana. This ER $\mathrm{Ca}^{2+}$ depletion followed by the elevation of cytosolic $\mathrm{Ca}^{2+}$ may trigger early signaling that leads to cell death. First, $\mathrm{Ca}^{2+}$ is required for protein processing and glycosylation, protein folding, and subunit assembly. The reduction of $\mathrm{ER} \mathrm{Ca}^{2+}$ levels results in unfolded protein response, an evolutionarily conserved stress response that can trigger apoptosis if ER dysfunction is severe or prolonged (Görlach et al. 2006; Shore et al. 2011). Second, the ER is a key organelle that initiates and modulates apoptosis. $\mathrm{ER} \mathrm{Ca}^{2+}$ release is an early signaling event for the initiation of apoptosis induced by many apoptotic signals (Pinton et al. 2008). The elevation of cytosolic $\mathrm{Ca}^{2+}$ may activate signaling cascades involved in celldeath-associated plant immune responses. For instance, an ERlocalized type IIB $\mathrm{Ca}^{2+}$-ATPase in $N$. benthamiana (NbCA1) functions in innate immunity-mediated programmed cell death, and downregulation of NbCA1 results in the modulation of intracellular calcium signaling in response to a cryptogein elicitor (Zhu et al. 2010). In our previous report, XA10 was found to form hexamers on the ER membrane, which may form a pore or affect existing ion pumps or channels on the ER to either suppress the ER $\mathrm{Ca}^{2+}$ intake or promote $\mathrm{ER} \mathrm{Ca}^{2+}$ release (Tian et al. 2014). In this study, both XA10-Ni and XA23-Ni showed self-interaction on the ER membrane. However, the details of how the XA10-like executor R proteins cause the disruption of the ER and the change of $\mathrm{Ca}^{2+}$ homeostasis in the ER remain to be investigated.

Members of Xa10-like gene family were physically mapped to the same locus or adjacent loci in the rice genome; among these members, $\mathrm{Xa23}-\mathrm{Ni}$ and $\mathrm{Xa23}$ encode an identical protein (Gu et al. 2008; Tian et al. 2014; Wang et al. 2014a, 2015). Xal0 was originally identified in Cas209, an indica subspecies of cultivated rice (genome type: AA) (Ogawa et al. 2003; Yoshimura et al. 1983). Xa23 was derived from wild rice $O$. rufipogon (genome type: AA) (Zhang et al. 2001). Xa10-Ni and Xa23-Ni are present in Nipponbare, a cultivar of the japonica subspecies (genome type: AA). The Xal0-like genes were also found to be present in other cultivated and wild rice species: B8BL96 from O. sativa subsp. indica (genome type: AA); BAT14641, Q2R1Y8, and Q2R1Z3 from $O$. sativa subsp. japonica (genome type: AA); J3KY37, J3N992, and J3N993 from O. brachyantha (genome type: FF); A0A0E0BKP3 from O. glumipatula (genome type: AA); A0A0E0F739 from O. meridionalis (genome type: AA); A0A0E0J3N1 from O. nivara (genome type: AA);
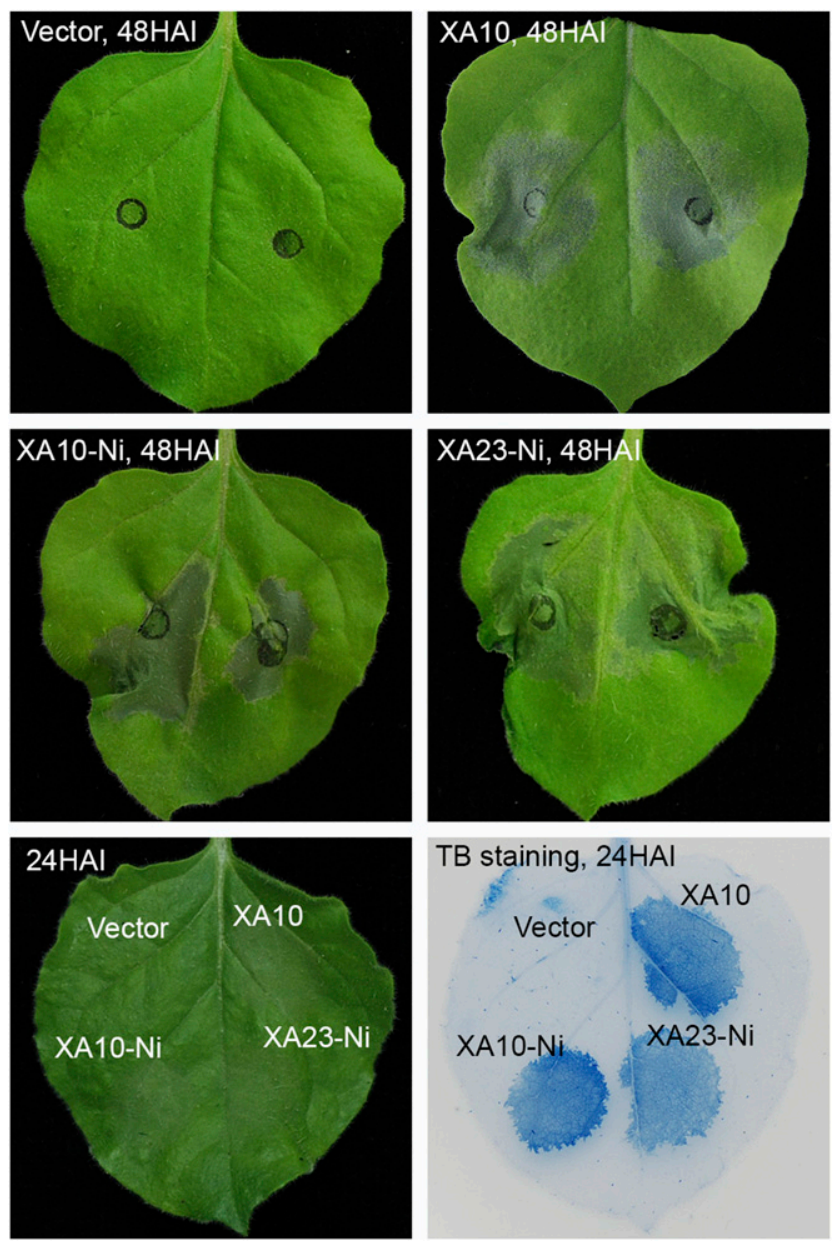

Fig. 4. XA10-Ni and XA23-Ni induced cell death in Nicotiana benthamiana. Images show phenotypes and trypan blue (TB) staining of $N$. benthamiana leaves transiently expressing empty vector pC1300 (Vector), XA10, XA10$\mathrm{Ni}$, or XA23-Ni at 24 or $48 \mathrm{~h}$ after infiltration (HAI) with Agrobacterium tumefaciens GV3101 harboring respective binary constructs. 
A0A0E0MGN7, A0A0E0MGN8, and A0A0E0MGN9 from O. punctata (genome type: BB); and A0A0E0RA89 and A0A0E0RA92 from $O$. rufipogon (genome type: AA) (Supplementary Figs. S11 and S12). The presence of Xa10-like genes in different genome types of rice and wild rice species suggests that Xa10-like genes might have originated from a single gene in an ancient rice genome before the divergence of wild and cultivated rice species. It was noted that $\mathrm{XaIO}-\mathrm{Ni}$ and $\mathrm{Xa23}-\mathrm{Ni}$ double knock-out mutants did not show any abnormal phenotype, indicating that, except for disease resistance to bacterial blight pathogens, the two Xa10-like genes in Nipponbare are dispensable for normal plant growth and development.

The specific recognition between $\mathrm{EBE}$ in the promoters of host target genes and the RVD in the central repeats of TAL effectors determines disease susceptibility or resistance. The coevolution between plants and pathogens leads to the generation of recessive insensitive alleles of $S$ genes in plants to avoid being targeted or the evolution of new TAL effectors in pathogens to overcome this avoidance (Antony et al. 2010; Iyer and McCouch 2004; Zhou et al. 2015). Similarly, the evolution of TAL effector-dependent $R$ genes is a kind of plant adaptation to the activity of TAL effectors as virulence factors. The mutation in the TAL effectors would enable pathogens to avoid being recognized by the EBE in the $R$ gene promoters and, therefore, overcome the TAL effector-dependent $R$-gene-mediated disease resistance. Among the four Xa10-like genes characterized, $X a 10$ and Xa23 confer race-specific disease resistance to field $X$. oryzae pv. oryzae strains that deliver AvrXa10 and AvrXa23, respectively, and the corresponding TAL effector binding sites, $E B E_{\mathrm{AvrXa10}}$ and $E B E_{\mathrm{AvrXa23}}$, were identified in $\mathrm{Xa10}$ and $\mathrm{Xa23}$ promoters (Hopkins et al. 1992; Wang et al. 2014b). However, neither $\mathrm{Xa10}-\mathrm{Ni}$ nor $\mathrm{Xa23}-\mathrm{Ni}$ in Nipponbare has been found to confer disease resistance to any field $X$. oryzae pv. oryzae strains or to be induced by any known natural TAL effector. It is likely that the rapid evolution of TAL effector genes in $X$. oryzae pv. oryzae has overcome TAL effector-dependent

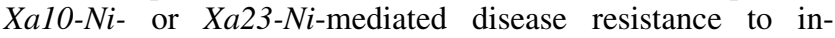
compatible bacterial strains (Vera Cruz et al. 2000). Nevertheless, the identification of $\mathrm{Xa1O}-\mathrm{Ni}$ and $\mathrm{Xa23-Ni}$ encoding functional executor $\mathrm{R}$ proteins provides opportunity to genetically generate novel disease resistance to Xanthomonas pathogens. One approach is to add multiple EBE, which match to the RVD of diffident TAL effectors from $X$. oryzae pv. oryzae or $X$. oryzae pv. oryzicola, to the promoters of Xa10-Ni or Xa23-Ni through transgenic or gene editing technology ( $\mathrm{Li}$ et al. 2016). The engineered $\mathrm{Xa10}-\mathrm{Ni}$ or $\mathrm{Xa23}-\mathrm{Ni}$ genes can provide broadspectrum and perhaps durable resistance to these pathogens. A similar approach has been successfully achieved in genetic
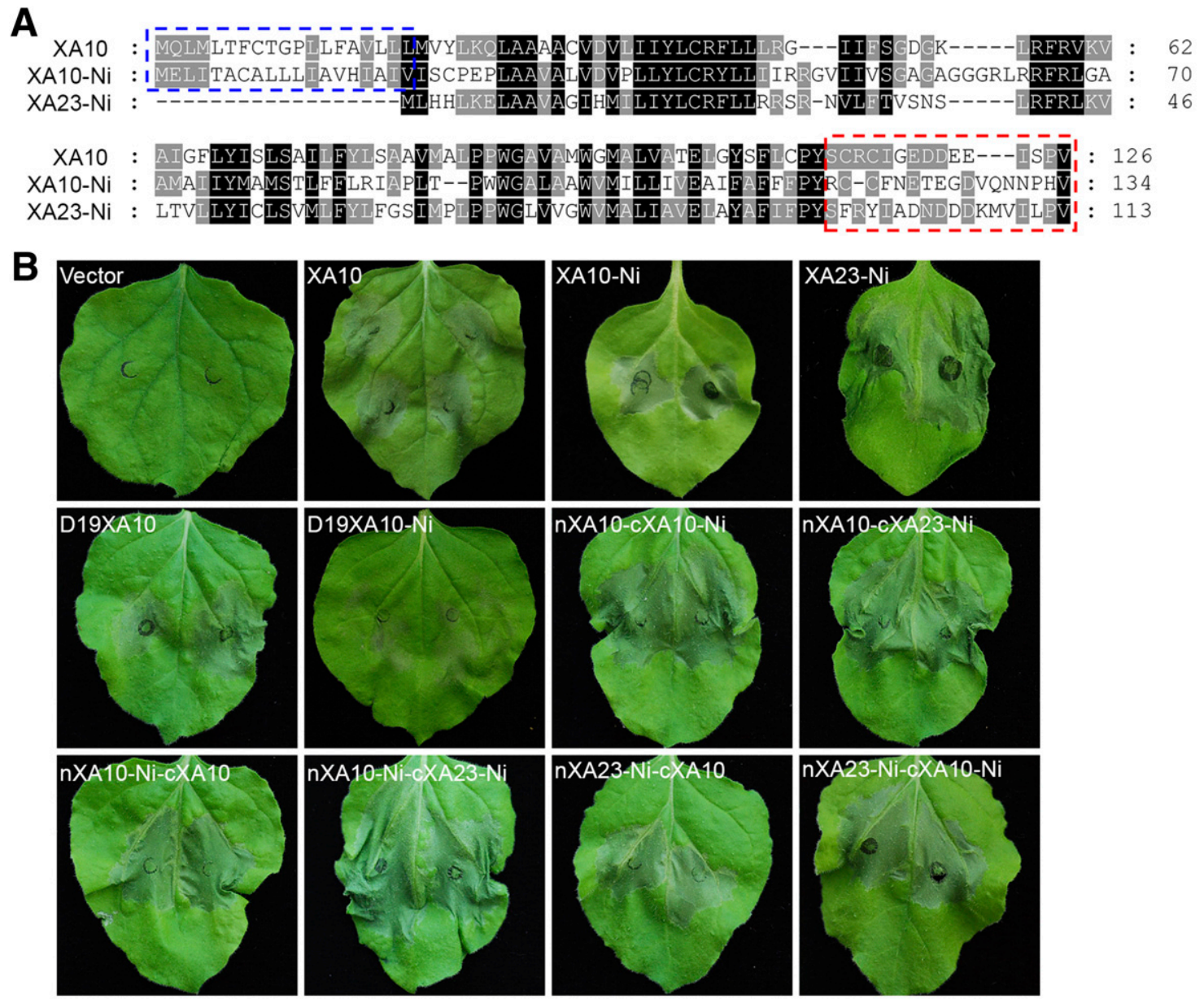

Fig. 5. Domain deletion and swapping between members of XA10-like proteins. A, Amino acid sequence alignment of XA10, XA10-Ni, and XA23-Ni. Amino acid sequences were aligned using the program Clustal W and shaded with GENEDOC software. Identical and conserved amino acids among three or two proteins are highlighted in black or gray backgrounds, respectively. N-terminal regions of XA10 and XA10-Ni in the blue box were deleted to generate Nterminal domain deletion variants D19XA10 and D19XA10-Ni, respectively. C-terminal regions of XA10, XA10-Ni, and XA23-Ni in the red box were swapped with each other to generate domain-swapping variants nXA10-cXA10-Ni, nXA10-cXA23-Ni, nXA10-Ni-cXA10, nXA10-Ni-cXA23-Ni, nXA23-NicXA10, and nXA23-Ni-cXA10-Ni. B, Phenotypes of Nicotiana benthamiana leaves transiently expressing empty vector pC1300 (Vector), members of XA10 proteins, and their mutants at $48 \mathrm{~h}$ after infiltration with Agrobacterium tumefaciens GV3101 harboring respective binary constructs. 
engineering of Xa10 and Xa27 promoters for broad-spectrum disease resistance to $X$. oryzae pv. oryzae or $X$. oryzae pv. oryzicola (Hummel et al. 2012; Zeng et al. 2015). Because the XA10-like proteins function in $N$. benthamiana, they may be used to engineer novel disease resistance in dicotyledonous plants to bacterial diseases caused by Xanthomonas strains that rely on TAL effectors for virulence.

\section{MATERIALS AND METHODS}

\section{Genes and constructs.}

The central repeats in dTALE-Xa10-Ni, dTALE-Xa23-Ni-1, dTALE-Xa23-Ni-2, TALEN 3F, and TALEN 4R were designed according to the codes between RVD and DNA nucleotides $(\mathrm{NI}=\mathrm{A}, \mathrm{NG}=\mathrm{T}, \mathrm{HD}=\mathrm{C}, \mathrm{NN} / \mathrm{NH}=\mathrm{G})$ (Boch et al. 2009). The central repeats in dTALE and TALEN genes were assembled and cloned into vector pTAL1 or pTAL3 according to the method described previously (Cermak et al. 2011). The SphI fragments of central DNA repeats in pTAL1-dTALE-Xa10-Ni, pTAL1dTALE-Xa23-Ni-1, and pTAL1-dTALE-Xa23-Ni-2 were isolated and used to replace the central DNA repeats of avrXa10 in pZWavrXa10 to generate pZWdTALE-Xa10-Ni, pZWdTALEXa23-Ni-1, and pZWdTALE-Xa23-Ni-2, respectively. These constructs were then digested with HindIII and inserted into the HindIII site of cosmid vector pHM1 (GenBank accession number EF059993) to generate pHM1dTALE-Xa10-Ni, pHM1dTALE-Xa23-Ni-1, and pHM1dTALE-Xa23-Ni-2 (Supplementary Table S4). The cosmid constructs were transferred into $X$. oryzae pv. oryzae strain $\mathrm{PXO} 99^{\mathrm{A}}$ by electroporation. The $B g l$ III-SacI fragment in pTAL3-3F, which contains the TALEN gene $3 F$, was isolated and cloned into pZH2Bik to generate pZH2Bik-3F. The HindIII-EcoRI fragment in pZH2Bik was isolated and cloned into pUC198AM to generate pUC198AM-HE. The $B g l I I-S a c$ I fragment in pTAL3-4R, which contains the TALEN gene $4 R$, was isolated and cloned into pUC198AM-HE to generate pUC198AM-HE-4R. The AscI-MluI fragment in pUC198AM-HE$4 \mathrm{R}$ was isolated and cloned into pZH2Bik-3F to generate final binary construct pZH2Bik-3F-4R. The expression cassette that contains target sequence in the coding region of $\mathrm{Xa23}-\mathrm{Ni}$ with sgRNA under the rice snoRNA U3 promoter in pYLsgRNA-OsU3 was amplified and cloned into vector pYLCRISPR/Cas $9 \mathrm{P}_{\mathrm{Ubi}^{-}} \mathrm{H}$ to generate binary construct pYLCas9-Xa23-Ni, according to the method described previously (Ma et al. 2015). The ER membrane marker for plant cells used in this study was eYFP-RcDGAT2, which was generated by fusion of eYFP with type 2 diacylglycerol acyltransferase from caster bean (Ricinus communis). The nYFP and CYFP genes that were used to fuse with $\mathrm{Xa10}-\mathrm{Ni}$ or $\mathrm{Xa23-Ni}$ genes for BiFC assay were reported previously (Citovsky et al. 2006). Other binary constructs were made based on CAMBIA vector pC1305.1. Binary constructs were introduced into A. tumefaciens stain AGL1 for rice transformation or GV3101 for infiltration of $N$. benthamiana.

\section{Rice cultivars and growth conditions.}

Rice (O. sativa L.) japonica Nipponbare was used in this study. All rice plants, including transgenic plants and plants

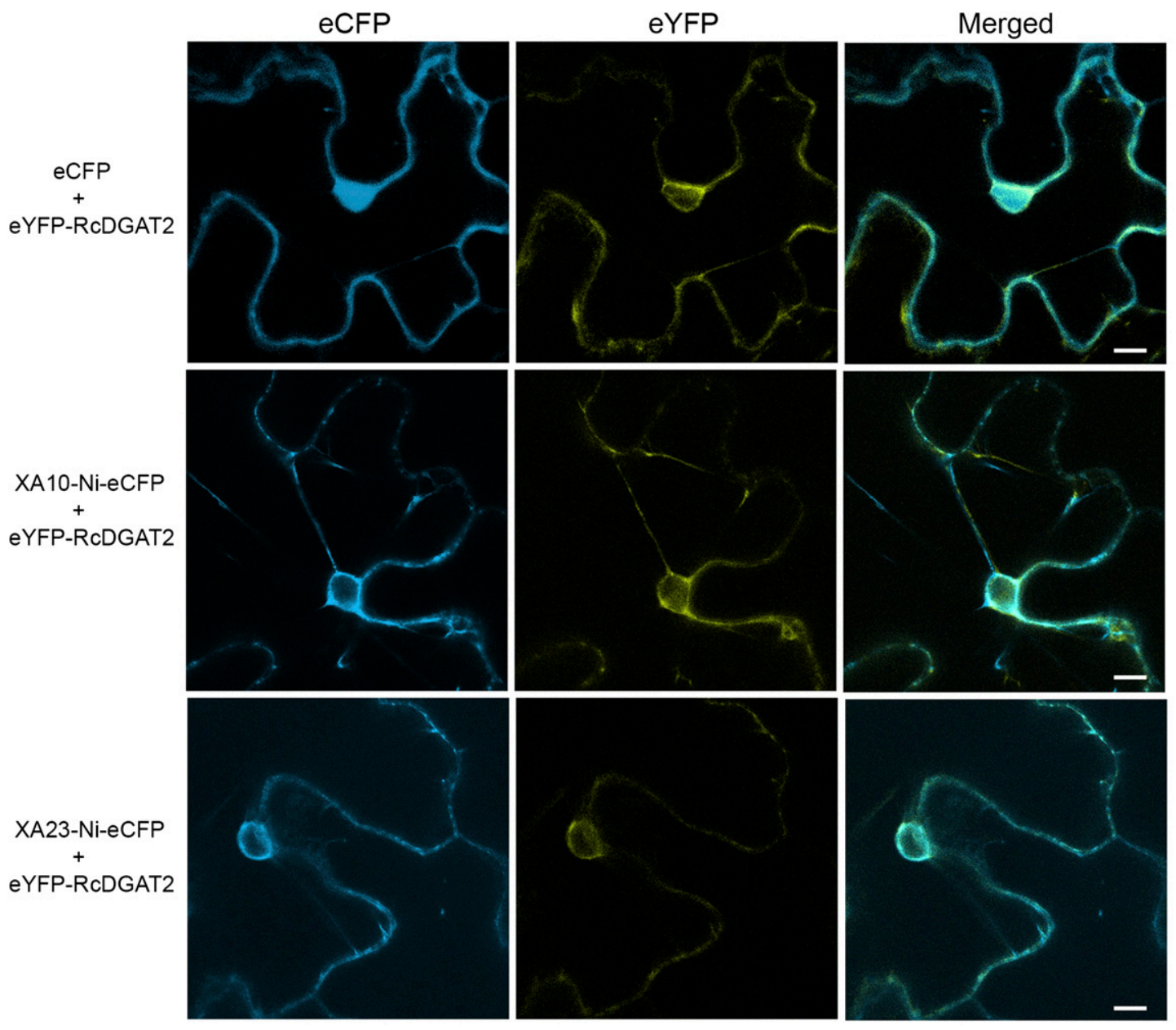

Fig. 6. Colocalization of eYFP-RcDGAT2 with XA10-Ni-eCFP or XA23-Ni-eCFP in leaf epidermal cells of Nicotiana benthamiana. Images were taken at $24 \mathrm{~h}$ after coinfiltration of $N$. benthamiana with Agrobacterium tumefaciens GV3101 harboring respective binary constructs. Bars $=10 \mu \mathrm{m}$. 
inoculated with $X$. oryzae pv. oryzae strains, were grown in greenhouse at $32^{\circ} \mathrm{C}$ for $12.5 \mathrm{~h}$ (light) and $25^{\circ} \mathrm{C}$ for $11.5 \mathrm{~h}$ (dark), with average humidity at $84 \%$.

\section{Rice transformation.}

Agrobacterium-mediated transformation of Nipponbare was performed according to the method described previously (Hiei et al. 1994).

\section{Bacterial blight inoculation.}

Bacterial blight inoculation was performed using the leafclipping method (Yang and White 2004), and disease scoring was measured as described previously (Gu et al. 2004). Experiments were repeated three times.

\section{5' and ' ${ }^{\prime}$ RACE and RT-PCR.}

The cDNA of $\mathrm{Xa10}-\mathrm{Ni}$ and $\mathrm{Xa23}-\mathrm{Ni}$ were isolated by $5^{\prime}$ and 3' RACE using a SMART RACE cDNA amplification kit (Clontech) as well as by RT-PCR. Both 5' RACE and 3' RACE were conducted in accordance with the manufacturer's instructions. The PCR products were cloned into pGEMT-easy vector (Promega Corp.) and sequenced. The specific primers for $5^{\prime}$ RACE and $3^{\prime}$ RACE of XalO-Ni were Xa10-Ni-5'-R (5'-ACA ATCGCAATATGGACGGC-3') and Xa10-Ni-3'-F (5'-CGAC
CCTCTTCTTCCTCCGCA-3'), respectively. The specific primers for 5' RACE and 3' RACE of $\mathrm{Xa23}-\mathrm{Ni}$ were $\mathrm{Xa} 23-\mathrm{Ni}-5^{\prime}-\mathrm{R}$ (5'-GATCATGTGTATACCGGCTACG-3') and Xa23-Ni-3'-F (5'-TCACCGTTTCCAACAGCCTCCG-3'), respectively. The specific primers for RT-PCR of $\mathrm{Xa10}-\mathrm{Ni}$ were Xa10-Ni-RT-F (5'-GCTTCTCCTCATCGCCGTCC-3') and Xa10-Ni-RT-R (5'GCGTGAGAGGAGCAATGCGG-3'). The specific primers for RT-PCR of $\mathrm{Xa23}-\mathrm{Ni}$ were Xa23-Ni-RT-F (5'-CGTAGCCGGT ATACACATGATC-3') and Xa23-Ni-RT-R (5'-CGACGAAGC AAGCAAAGCTGGC- $3^{\prime}$ ).

\section{qRT-PCR.}

qRT-PCR was performed in accordance with the procedures described previously ( $\mathrm{Gu}$ et al. 2011). The expression of the rice ubiquitin gene 5 (UBQ5) was used as the internal control. The specific primer pairs for $\mathrm{Xa10}-\mathrm{Ni}, \mathrm{Xa23}-\mathrm{Ni}$, and $U B Q 5$ were Xa10-Ni-Q-F (5'-CGACCCTCTTCTTCCTCCGCA-3')/Xa10-NiQ-R (5'-TGTACCATCTGATGAGCGCGAG-3'), Xa23-Ni-Q-F (5'-GTAGCCGGTATACACATGATCCTC-3')/Xa23-Ni-Q-R (5'GTCTCTCGGTCATGCTGTTCTAC-3'), and UBQ5-F (5'-AAC CACTTCGACCGCCACT-3')/UBQ5-R (5'-GTTCGATTTCCTC CTCCTTCC-3'), respectively. The qRT-PCR experiments were performed in triplicate and the data are presented as means \pm standard deviation.

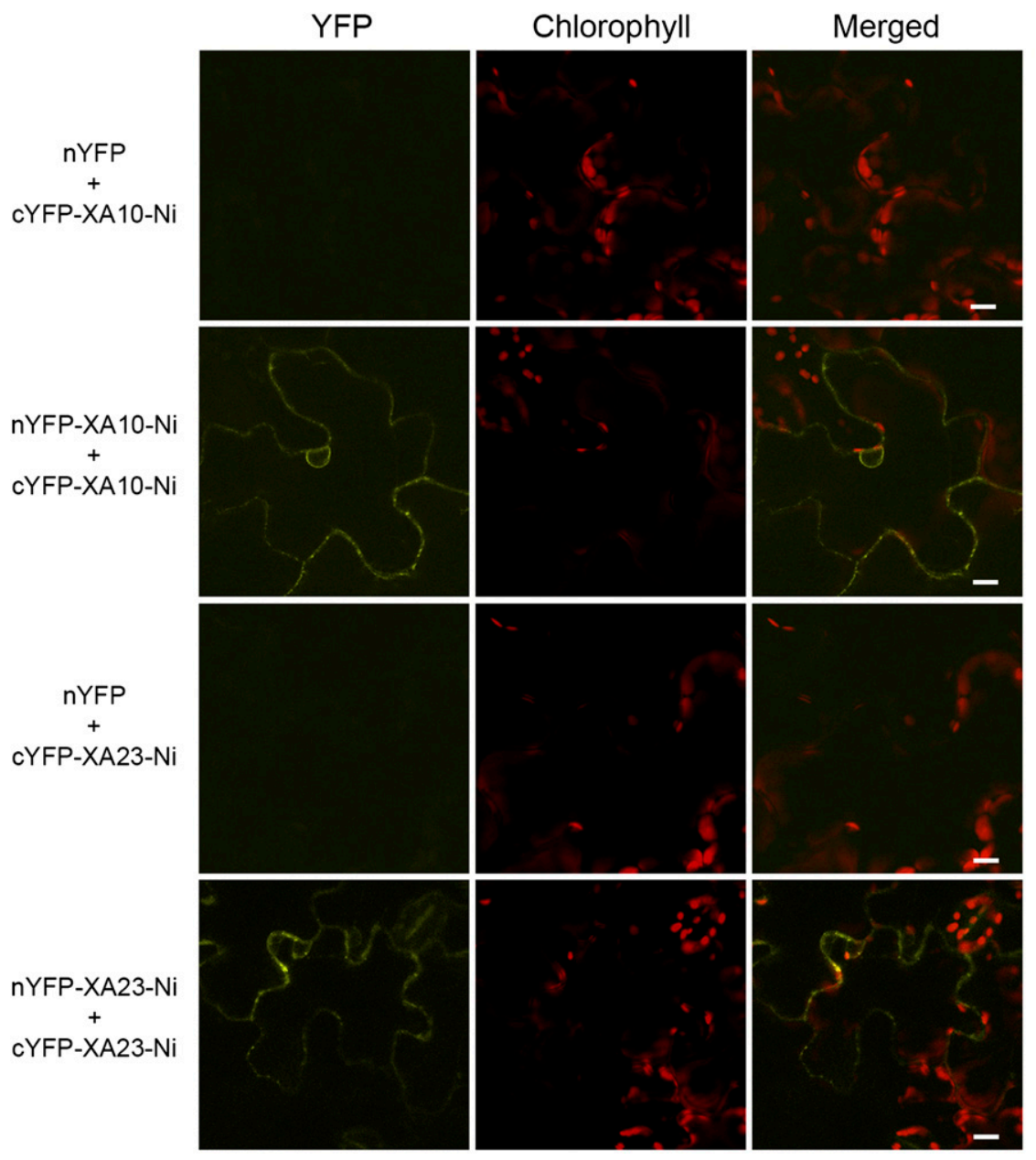

Fig. 7. Detection of self-interaction of XA10-like proteins in leaf epidermal cells of Nicotiana benthamiana by bimolecular fluorescence complementation. Representative images from confocal laser-scanning microscopy showed reconstituted yellow fluorescent protein fluorescence (YFP), chlorophyll autofluorescence (Chlorophyll), and merged channels (Merged) in leaf epidermal cells of $N$. benthamiana transiently coexpressing $\mathrm{nYFP}-\mathrm{XA10-Ni}$ and cYFPXA10-Ni or nYFP-XA23-Ni and cYFP-XA23-Ni at $24 \mathrm{~h}$ after coinfiltration with Agrobacterium tumefaciens harboring corresponding binary constructs. The coexpression of nYFP with cYFP-XA10-Ni or cYFP-XA23-Ni serves as negative controls. Bars $=10 \mu \mathrm{m}$. 

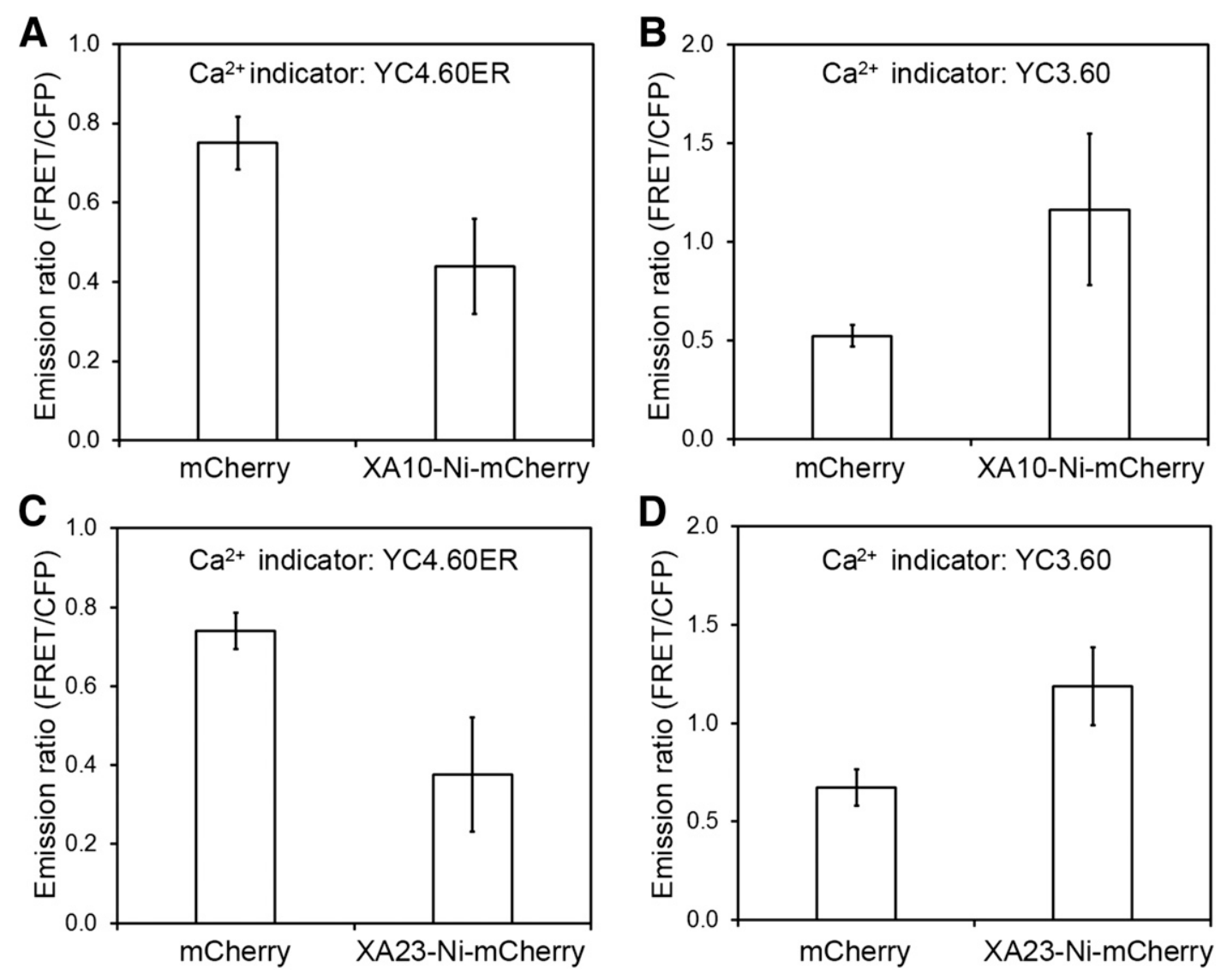

Fig. 8. XA10-Ni and XA23-Ni induced calcium depletion from the endoplasmic reticulum (ER) in leaf epidermal cells of Nicotiana benthamiana. A, Average emission ratios (fluorescence resonance energy transfer/cyan fluorescent protein [FRET/CFP]) of YC4.60ER in leaf epidermal cells of $N$. benthamiana transiently coexpressing YC4.60ER with mCherry (cell numbers measured: $n=28$ ) or XA10-Ni-mCherry $(n=31)$. B, Average emission ratios (FRET/CFP) of YC3.60 in leaf epidermal cells of $N$. benthamiana transiently coexpressing YC3.60 with mCherry $(n=20)$ or XA10-Ni-mCherry $(n=12)$. C, Average emission ratios (FRET/CFP) of YC4.60ER in leaf epidermal cells of $N$. benthamiana transiently coexpressing YC4.60ER with mCherry $(n=11)$ or XA23-Ni-mCherry $(n=16)$. D, Average emission ratios (FRET/CFP) of YC3.60 in leaf epidermal cells of $N$. benthamiana transiently coexpressing YC3.60 with mCherry $(n=20)$ or XA23-Ni-mCherry $(n=17)$.

\section{Infiltration of $N$. benthamiana \\ with Agrobacterium and confocal microscopy.}

Suspensions of Agrobacterium strain GV3101 harboring binary constructs were infiltrated into leaves of $N$. benthamiana as described previously (Kay et al. 2007). The infiltrated plants were grown under $16 \mathrm{~h}$ of light and $8 \mathrm{~h}$ of darkness at $25^{\circ} \mathrm{C}$. Phenotype was checked at 24 to 48 HAI. For confocal microscopy, water-mounted leaf sections were examined using an LSM 510 Exciter Upright confocal microscope system (Carl Zeiss). The excitation/emission combinations for eCFP, eYFP, mCherry, and autofluorescence of chlorophyll were band passes of $405 / 475$ to $525,514 / 560$ to $615,543 / 600$ to 650 , and $514 / 610 \mathrm{~nm}$, respectively.

\section{Trypan blue staining.}

Cell death in plant tissues was detected by trypan blue staining in accordance with the procedures described previously (Tian et al. 2014).

\section{Measurement of calcium in leaf cells of $N$. benthamiana.}

The free $\mathrm{Ca}^{2+}$ concentration in the ER and cytoplasm of leaf cells of $N$. benthamiana was measured with $\mathrm{Ca}^{2+}$ indicators YC4.60ER and YC3.60 using the method described previously (Iwano et al. 2009). For confocal microscopy, N. benthamiana cells at 20 to 30 HAI were imaged for mCherry, eCFP, eYFP, and FRET using an LSM 510 Exciter Upright confocal microscope system (Carl Zeiss). The excitation/emission combinations for mCherry, eCFP, eYFP, and autofluorescence of chlorophyll were band passes of $543 / 600$ to $650,458 / 475$ to $525,488 / 505$ to 530 , and $458 / 505$ to $530 \mathrm{~nm}$, respectively. Images analysis and FRET ratio calculation were conducted using the methods described previously (Tian et al. 2014).

\section{ACKNOWLEDGMENTS}

We thank Y. Liu for providing plasmids pYLsgRNA-OsU3 and pYLCRISPR/Cas9P $\mathrm{Ubi}-\mathrm{H}$, D. Voytas for the Golden Gate TAL effector kit (through Addgene), and K. H. Ong for critical reading of the manuscript. This research is supported by the National Research Foundation, Prime Minister's Office, Singapore under its Competitive Research Programme (NRF-CRP7-2010-02) and administered by Temasek Life Sciences and National University of Singapore.

\section{LITERATURE CITED}

Antony, G., Zhou, J., Huang, S., Li, T., Liu, B., White, F., and Yang, B. 2010. Rice $x a 13$ recessive resistance to bacterial blight is defeated by induction of the disease susceptibility gene $O s-11 N 3$. Plant Cell 22: 3864-3876.

Boch, J., Scholze, H., Schornack, S., Landgraf, A., Hahn, S., Kay, S., Lahaye, T., Nickstadt, A., and Bonas, U. 2009. Breaking the code of DNA binding specificity of TAL-type III effectors. Science 326: 1509-1512.

Bogdanove, A. J., Schornack, S., and Lahaye, T. 2010. TAL effectors: Finding plant genes for disease and defense. Curr. Opin. Plant Biol. 13: 394-401. 
Cermak, T., Doyle, E. L., Christian, M., Wang, L., Zhang, Y., Schmidt, C., Baller, J. A., Somia, N. V., Bogdanove, A. J., and Voytas, D. F. 2011. Efficient design and assembly of custom TALEN and other TAL effectorbased constructs for DNA targeting. Nucleic Acids Res. 39:e82.

Cernadas, R. A., Doyle, E. L., Niño-Liu, D. O., Wilkins, K. E., Bancroft, T., Wang, L., Schmidt, C. L., Caldo, R., Yang, B., White, F. F., Nettleton, D., Wise, R. P., and Bogdanove, A. J. 2014. Code-assisted discovery of TAL effector targets in bacterial leaf streak of rice reveals contrast with bacterial blight and a novel susceptibility gene. PLoS Pathog. 10: e1003972.

Chen, L.-Q., Hou, B.-H., Lalonde, S., Takanaga, H., Hartung, M. L., Qu, X.-Q., Guo, W.-J., Kim, J.-G., Underwood, W., Chaudhuri, B., Chermak, D., Antony, G., White, F. F., Somerville, S. C., Mudgett, M. B., and Frommer, W. B. 2010. Sugar transporters for intercellular exchange and nutrition of pathogens. Nature 468:527-532.

Chu, Z., Yuan, M., Yao, J., Ge, X., Yuan, B., Xu, C., Li, X., Fu, B., Li, Z., Bennetzen, J. L., Zhang, Q., and Wang, S. 2006. Promoter mutations of an essential gene for pollen development result in disease resistance in rice. Genes Dev. 20:1250-1255.

Citovsky, V., Lee, L.-Y., Vyas, S., Glick, E., Chen, M.-H., Vainstein, A., Gafni, Y., Gelvin, S. B., and Tzfira, T. 2006. Subcellular localization of interacting proteins by bimolecular fluorescence complementation in planta. J. Mol. Biol. 362:1120-1131.

Görlach, A., Klappa, P., and Kietzmann, T. 2006. The endoplasmic reticulum: Folding, calcium homeostasis, signaling, and redox control. Antioxid. Redox Signaling 8:1391-1418.

Gu, K., Chiam, H., Tian, D., and Yin, Z. 2011. Molecular cloning and expression of heteromeric ACCase subunit genes from Jatropha curcas. Plant Sci. 180:642-649.

Gu, K., Sangha, J. S., Li, Y., and Yin, Z. 2008. High-resolution genetic mapping of bacterial blight resistance gene Xa10. Theor. Appl. Genet. 116:155-163.

Gu, K., Tian, D., Yang, F., Wu, L., Sreekala, C., Wang, D., Wang, G. L., and Yin, Z. 2004. High-resolution genetic mapping of Xa27(t), a new bacterial blight resistance gene in rice, Oryza sativa L. Theor. Appl. Genet. 108:800-807.

Gu, K., Yang, B., Tian, D., Wu, L., Wang, D., Sreekala, C., Yang, F., Chu, Z., Wang, G.-L., White, F. F., and Yin, Z. 2005. $R$ gene expression induced by a type-III effector triggers disease resistance in rice. Nature 435 : $1122-1125$

Hiei, Y., Ohta, S., Komari, T., and Kumashiro, T. 1994. Efficient transformation of rice (Oryza sativa L.) mediated by Agrobacterium and sequence analysis of the boundaries of the T-DNA. Plant J. 6:271-282.

Hopkins, C. M., White, F. F., Choi, S. H., Guo, A., and Leach, J. E. 1992. Identification of a family of avirulence genes from Xanthomonas oryzae pv. oryzae. Mol. Plant-Microbe Interact. 5:451-459.

Hu, Y., Zhang, J., Jia, H., Sosso, D., Li, T., Frommer, W. B., Yang, B., White, F. F., Wang, N., and Jones, J. B. 2014. Lateral organ boundaries 1 is a disease susceptibility gene for citrus bacterial canker disease. Proc. Natl. Acad. Sci. U.S.A. 111:E521-E529.

Huang, S., Antony, G., Li, T., Liu, B., Obasa, K., Yang, B., and White, F. F. 2016. The broadly effective recessive resistance gene $x a 5$ of rice is a virulence effector-dependent quantitative trait for bacterial blight. Plant J. 86:186-194.

Hummel, A. W., Doyle, E. L., and Bogdanove, A. J. 2012. Addition of transcription activator-like effector binding sites to a pathogen strain-specific rice bacterial blight resistance gene makes it effective against additional strains and against bacterial leaf streak. New Phytol. 195:883-893.

Hutin, M., Sabot, F., Ghesquière, A., Koebnik, R., and Szurek, B. 2015. A knowledge-based molecular screen uncovers a broad-spectrum OsSWEET14 resistance allele to bacterial blight from wild rice. Plant J. 84:694-703.

Iwano, M., Entani, T., Shiba, H., Kakita, M., Nagai, T., Mizuno, H., Miyawaki, A., Shoji, T., Kubo, K., Isogai, A., and Takayama, S. 2009. Fine-tuning of the cytoplasmic $\mathrm{Ca}^{2+}$ concentration is essential for pollen tube growth. Plant Physiol. 150:1322-1334.

Iyer, A. S., and McCouch, S. R. 2004. The rice bacterial blight resistance gene $x a 5$ encodes a novel form of disease resistance. Mol. Plant-Microbe Interact. 17:1348-1354.

Kay, S., Hahn, S., Marois, E., Hause, G., and Bonas, U. 2007. A bacterial effector acts as a plant transcription factor and induces a cell size regulator. Science 318:648-651.

Li, J., Meng, X., Zong, Y., Chen, K., Zhang, H., Liu, J., Li, J., and Gao, C. 2016. Gene replacements and insertions in rice by intron targeting using CRISPR-Cas9. Nat. Plants 2:Article 16139.

Li, T., Huang, S., Zhou, J., and Yang, B. 2013. Designer TAL effectors induce disease susceptibility and resistance to Xanthomonas oryzae pv. oryzae in rice. Mol. Plant 6:781-789.
Li, T., Liu, B., Spalding, M. H., Weeks, D. P., and Yang, B. 2012. Highefficiency TALEN-based gene editing produces disease-resistant rice. Nat. Biotechnol. 30:390-392.

Ma, X., Zhang, Q., Zhu, Q., Liu, W., Chen, Y., Qiu, R., Wang, B., Yang, Z., Li, H., Lin, Y., Xie, Y., Shen, R., Chen, S., Wang, Z., Chen, Y., Guo, J., Chen, L., Zhao, X., Dong, Z., and Liu, Y.-G. 2015. A robust CRISPR/Cas9 system for convenient, high-efficiency multiplex genome editing in monocot and dicot plants. Mol. Plant 8:1274-1284.

Moscou, M. J., and Bogdanove, A. J. 2009. A simple cipher governs DNA recognition by TAL effectors. Science 326:1501.

Niño-Liu, D. O., Ronald, P. C., and Bogdanove, A. J. 2006. Xanthomonas oryzae pathovars: Model pathogens of a model crop. Mol. Plant Pathol. 7:303-324.

Ogawa, T., Endo, N., Busto, G. A., Jr., Tabien, R. E., Taura, S., Khush, G. S., Brar, D. S., and Hardy, B. 2003. Evolutionary significance of varietal groups resistant to bacterial leaf blight in rice. Pages 99-103 in: Advances in Rice Genetics. G. S. Khush, D. S. Brar, and B. Hardy, eds. International Rice Research Institute, Los Baños, Philippines.

Pinton, P., Giorgi, C., Siviero, R., Zecchini, E., and Rizzuto, R. 2008. Calcium and apoptosis: ER-mitochondria $\mathrm{Ca}^{2+}$ transfer in the control of apoptosis. Oncogene 27:6407-6418.

Römer, P., Hahn, S., Jordan, T., Strauss, T., Bonas, U., and Lahaye, T. 2007. Plant pathogen recognition mediated by promoter activation of the pepper Bs3 resistance gene. Science 318:645-648.

Shore, G. C., Papa, F. R., and Oakes, S. A. 2011. Signaling cell death from the endoplasmic reticulum stress response. Curr. Opin. Cell Biol. 23: 143-149.

Strauss, T., van Poecke, R. M., Strauss, A., Römer, P., Minsavage, G. V., Singh, S., Wolf, C., Strauss, A., Kim, S., Lee, H.-A., Yeom, S. I., Parniske, M., Stall, R. E., Jones, J. B., Choi, D., Prins, M., and Lahaye, T. 2012. RNA-seq pinpoints a Xanthomonas TAL-effector activated resistance gene in a large-crop genome. Proc. Natl. Acad. Sci. U.S.A. 109:19480-19485.

Streubel, J., Pesce, C., Hutin, M., Koebnik, R., Boch, J., and Szurek, B. 2013. Five phylogenetically close rice SWEET genes confer TAL effector-mediated susceptibility to Xanthomonas oryzae pv. oryzae. New Phytol. 200:808-819

Sugio, A., Yang, B., Zhu, T., and White, F. F. 2007. Two type III effector genes of Xanthomonas oryzae pv. oryzae control the induction of the host genes $O s T F I I A \gamma 1$ and $O s T F X 1$ during bacterial blight of rice. Proc. Natl. Acad. Sci. U.S.A. 104:10720-10725.

Tian, D., Wang, J., Zeng, X., Gu, K., Qiu, C., Yang, X., Zhou, Z., Goh, M., Luo, Y., Murata-Hori, M., White, F. F., and Yin, Z. 2014. The rice TAL effector-dependent resistance protein XA10 triggers cell death and calcium depletion in the endoplasmic reticulum. Plant Cell 26:497-515.

Vera Cruz, C. M., Bai, J., Oña, I., Leung, H., Nelson, R. J., Mew, T.-W., and Leach, J. E. 2000. Predicting durability of a disease resistance gene based on an assessment of the fitness loss and epidemiological consequences of avirulence gene mutation. Proc. Natl. Acad. Sci. U.S.A. 97: 13500-13505.

Wang, C., Fan, Y., Zheng, C., Qin, T., Zhang, X., and Zhao, K. 2014a. Highresolution genetic mapping of rice bacterial blight resistance gene $\mathrm{Xa23}$. Mol. Genet. Genomics 289:745-753.

Wang, C., Zhang, X., Fan, Y., Gao, Y., Zhu, Q., Zheng, C., Qin, T., Li, Y., Che, J., Zhang, M., Yang, B., Liu, Y., and Zhao, K. 2015. XA23 is an executor $\mathrm{R}$ protein and confers broad-spectrum disease resistance in rice. Mol. Plant 8:290-302.

Wang, C.-L., Qin, T.-F., Yu, H.-M., Zhang, X. P., Che, J.-Y., Gao, Y., Zheng, C.-K., Yang, B., and Zhao, K.-J. 2014b. The broad bacterial blight resistance of rice line $\mathrm{CBB} 23$ is triggered by a novel transcription activator-like (TAL) effector of Xanthomonas oryzae pv. oryzae. Mol. Plant Pathol. 15:333-341

Wu, L., Goh, M. L., Sreekala, C., and Yin, Z. 2008. XA27 depends on an amino-terminal signal-anchor-like sequence to localize to the apoplast for resistance to Xanthomonas oryzae pv. oryzae. Plant Physiol. 148: 1497-1509.

Yang, B., Sugio, A., and White, F. F. 2006. Os8N3 is a host diseasesusceptibility gene for bacterial blight of rice. Proc. Natl. Acad. Sci. U.S.A 103:10503-10508.

Yang, B., and White, F. F. 2004. Diverse members of the AvrBs3/PthA family of type III effectors are major virulence determinants in bacterial blight disease of rice. Mol. Plant-Microbe Interact. 17:1192-1200.

Yoshimura, A., Mew, T. W., Khush, G. S., and Omura, T. 1983. Inheritance of resistance to bacterial blight in rice cultivar Cas 209. Phytopathology 73:1409-1412.

Yu, Y., Streubel, J., Balzergue, S., Champion, A., Boch, J., Koebnik, R., Feng, J., Verdier, V., and Szurek, B. 2011. Colonization of rice leaf blades by an African strain of Xanthomonas oryzae pv. oryzae depends 
on a new TAL effector that induces the rice nodulin-3 Os11N3 gene. Mol. Plant-Microbe Interact. 24:1102-1113.

Zeng, X., Tian, D., Gu, K., Zhou, Z., Yang, X., Luo, Y., White, F. F., and Yin, Z. 2015. Genetic engineering of the Xa10 promoter for broadspectrum and durable resistance to Xanthomonas oryzae pv. oryzae. Plant Biotechnol. J. 13:993-1001.

Zhang, J., Yin, Z., and White, F. 2015. TAL effectors and the executor $R$ genes. Front. Plant Sci. 6:641.

Zhang, Q., Wang, C., Zhao, K., Zhao, Y., Caslana, V. C., Zhu, X., Li, D., and Jiang, Q. 2001. The effectiveness of advanced rice lines with new resistance gene $X a 23$ to rice bacterial blight. Rice Genet. Newsl. 18: 71-72.

Zhou, J., Peng, Z., Long, J., Sosso, D., Liu, B., Eom, J. S., Huang, S., Liu, S., Vera Cruz, C., Frommer, W. B., White, F. F., and Yang, B. 2015. Gene targeting by the TAL effector PthXo 2 reveals cryptic resistance gene for bacterial blight of rice. Plant J. 82:632-643.

Zhu, X., Caplan, J., Mamillapalli, P., Czymmek, K., and Dinesh-Kumar, S. P. 2010. Function of endoplasmic reticulum calcium ATPase in innate immunity-mediated programmed cell death. EMBO J. 29:10071018. 